\title{
Bifurcation from Simple Eigenvalue for Functional-Differential Equations
}

\author{
E. Stepanov
}

\begin{abstract}
A method to determine bifurcation points of complicated boundary value problems for functional-differential equations is developed, which provides sufficient conditions on existence of intervals containing bifurcation points in terms of some simple estimates rather than in terms of spectral properties of the linearized problem. In the case of Frechèt-differentiable non-linearity the method reduces to the study of bifurcation from simple eigenvalues of the linearized problem. It still works, if the linearized problem contains homogeneous but nonlinear operators. The heart of the approach consists in the study of branching equations obtained by Lyapunov-Schmidt reduction and a special non-equivalent change of variables. Construction of such equations based on the choice of generalized Green's operator is discussed. At last, some applications to second order equations with deviating argument are provided.
\end{abstract}

Keywords: Functional-differential equations, bifurcation, topological degree, linearization AMS subject classification: 34 A 47, $34 \mathrm{~K} 10 ; 58$ F 14, 34 B 27

\section{Introduction}

Consider the following simple nonlinear boundary value problem for a second order functional-differential equation with deviating argument of the unknown $x \in A C^{1,1}$ from the space $A C^{1,1}=A C^{1,1}(0, T)$ of functions with absolutely continuous first derivative and second derivative from the Lebesgue space $L^{1}(0, T)$ :

$$
\left\{\begin{array}{rlrl}
\ddot{x}+\left(\lambda_{1}+q(t)\right) x(h(t)) & =r(x) & & (t \in[0, T]), \\
x(\tau) & =0 \quad & (\tau \notin[0, T]), \\
x(0)-x(T) & =\phi_{1}(x), & \\
\dot{x}(0)-\dot{x}(T) & =\phi_{2}(x), &
\end{array}\right.
$$

where $T>0$ is given, $q \in L^{1}(0, T), h:[0, T] \rightarrow \mathbb{R}$ is measurable, $r: A C^{1,1} \rightarrow L^{1}(0, T)$ and $\phi_{1}, \phi_{2}: A C^{1,1} \rightarrow \mathbb{R}$ are highly nonlinear operators satisfying $r(0)=0$ and $\phi_{i}(0)=$ $0 \quad(i=1,2)$, while $\lambda_{1} \in \mathbb{R}$ is a bifurcation parameter. It is clear that this problem admits a trivial solution $x \equiv 0$. One is interested however in finding those values of the parameter $\lambda_{1}$ (called bifurcation points) that give rise to branches of non-trivial

E. Stepanov: Scuola Normale Superiore, Piazza dei Cavalieri 7, 56126 Pisä, Italy; e-mail: stepanov@cibs.sns.it 
solutions. We note that the question of finding constructive methods to study solvability of similar linear eigenvalue problems raised recently in the paper of $V$. Maksimov and A. Rumyantsev [12].

It is well-known that under rather unrestrictive conditions of smoothness of the operators all the bifurcation points of problem (1) provide non-trivial solvability of the respective linearized problem

$$
\left\{\begin{aligned}
\ddot{x}+\left(\lambda_{1}+q(t)\right) x(h(t)) & =0 \quad(t \in[0, T]), \\
x(\tau) & =0 \quad(\tau \notin[0, T]), \\
x(0) & =x(T) \\
\dot{x}(0) & =\dot{x}(T),
\end{aligned}\right.
$$

in other words, they are characteristic values of the latter. Thus to solve the posed bifurcation problem it is reasonable first to find all characteristic values of problem (2) and then to check each of the latter, whether it is really a bifurcation point of problem (1). Such a classical scheme can be easily established for many certain simple ordinary (or partial) differential equations. However, this is evidently not the case in the above posed problem. First, (2) is too complicated to be solved explicitly and hence even the existence and location of its characteristic values (i.e. points "suspectable" for bifurcation) constitute non-trivial problems. What is more, although one has a whole variety of classical results starting from the simple eigenvalue theorem of $M$. $G$. Crandall and P. Rabinowitz [5] or a linearization theorem of M. A. Krasnosel'skii [11: Theorem 56.4], to verify whether a characteristic value of (2) is actually a bifurcation point of (1) one needs usually deeper knowledge of the structure of the solution set of the linearized problem to apply them, like the multiplicities of the characteristic values. Such an information is clearly difficult to obtain.

We emphasize that it is a typical situation in classical bifurcation methods (see $[4$, 10] for a general overview) that one needs firstly points "suspectable" for bifurcation and secondly some more information about the latter formulated usually in terms of certain multiplicities. This applies also to the most general bifurcation results obtained by P.M. Fitzpatrick and J. Pejsachovicz, which use the topological invariant (called parity) of the parametrized families of Fredholm maps "responsible" for the bifurcation phenomena. However, the difficulty in computing the parity in applications [6, 7] is again reduced to checking the multiplicity of the characteristic values of the respective linearized problem.

In this paper, to handle the bifurcation problems for rather general functionaldifferential equations, including the ones of type (1), we develop a very simple method originally presented by S. A. Vavilov in [21] and then applied with some modifications in $[19,22]$ to the analysis of non-trivial solvability of various boundary value problems. The general advantage of this method consists in the fact that it allows to formulate sufficient conditions on the existence of bifurcation points only in terms of the solvability of some systems of algebraic inequalities. In practice (e.g., in applications to functionaldifferential equations) such conditions can always be easily verified, unlike conditions involving spectral properties of the linearized problem, which can be effectively verified 
only if the latter has not too complicated structure. Hence, this approach seems to be particularly adapted for such problems. Besides, from the algorithmical point of view, it consists of several rather straightforward steps, many of which can even be performed by some computer programs doing analytic calculations because in practice they involve only symbolic substitutions. The second advantage of the method is that it still works in somewhat less classical cases dealing with linearizations leading to problems which are homogeneous of order one while being not linear. In such situations it is impossible to refer to the linear spectral theory. Applications of this kind frequently appear in functional-differential equations with "maxima".

In principle, the method used in this paper allows not only to localize the intervals containing the bifurcation points, but also to apply some numerical approximation techniques to find both the bifurcation points and the branches of non-trivial solutions. However, we pay a high price for such a constructivity of the approach. In fact, it will be shown that in most important cases this method leads only to analogies of the simple eigenvalue theorem, and hence, from the abstract point of view, is much less general than the above-mentioned classical results.

The heart of the approach consists of special linearization theorems extending their prototype claimed in [23] and based on the application of a standard Lyapunov-Schmidt reduction, the special non-equivalent substitution of variables and the Leray-Schauder topological degree theory to analyze the solvability of systems of infinte-dimensional branching equations. We mention that each of these instruments, both separately and in combinations, is more or less customary in general bifurcation theory. A very similar approach combining these methods in the same way as we do here (only the nonequivalent substitution was different) has been successfully used recently by M. Martelli in solving so-called cobifurcation (atypical bifurcation) problems in [13]. It is interesting to note that the results also of "simple eigenvalue" character, which seem to be rather close to ours, have been obtained in bifurcation theory for differential equations on manifolds by M. Furi, M. Martelli and M.P. Pera (see [8] and the references therein). However, in most papers devoted to local bifurcation an attempt is made to find bifurcation points explicitly, while due to the complexity of the problems we are only able to prove their existence inside some regions (and then, if necessary, try to find them numerically). Thus, some results of global bifurcation seem to be more in this spirit (see, e.g., [9: Theorem 2.2]), although in this paper we deal only with local phenomena. The reader will notice that the results we present here have common background with the general continuation principles introduced and elaborated by J. Mawhin (for an uptodate survey see [14]).

Our interest lies mainly in applications to functional-differential equations. In fact, we will show how by means of the appropriate choice of the generalized Green operator and the respective construction of branching equations it is possible to formulate sufficient conditions on the existence of bifurcation points for rather complex functionaldifferential equations in terms of rather sharp estimates sensible to the presence of nonlocal operators in the problem. Moreover, we will show how to construct such estimates in a simple and straightforward way, independently of the particular structure of the chosen solution space. 


\section{Abstract problem formulation and preliminaries}

In the sequel we study bifurcation problems for some functional-differential equations of the form

$$
\left\{\begin{array}{c}
\mathcal{L} x=\mathcal{F}\left(x, \lambda_{1}\right), \\
l x=\phi\left(x, \lambda_{1}\right),
\end{array}\right.
$$

where $x \in E_{1}$ is the unknown element inside a real Banach space $E_{1}$ of sufficiently "regular" functions, $\lambda_{1} \in \mathbb{R}$ is a bifurcation parameter, $\mathcal{L}: E_{1} \rightarrow E$ is a linear (densely defined) operator, $\mathcal{F}: E_{1} \times \mathbb{R} \rightarrow E$ is a nonlinear operator such that $\mathcal{F}\left(0, \lambda_{1}\right)=0$ for all $\lambda_{1}, l: E_{1} \rightarrow \mathbb{R}^{n}$ and $\phi: E_{1} \times \mathbb{R} \rightarrow \mathbb{R}^{n}$ are linear and nonlinear vector functionals, respectively, representing the boundary conditions. Moreover, let $\phi\left(0, \lambda_{1}\right)=0$ for all $\lambda_{1}$ and $E_{1} \simeq E \times \mathbb{R}^{n}$, where $E$ is a real infinite-dimensional Banach space and $\simeq$ means isomorphy.

It is well-known [1: Chapter 6] that if $E$ is a space of summable functions, like Lebesgue or Orlicz space, the above setting covers a wide range of problems in the theory of functional-differential equations. In each case, the respective class of equations is determined by the choice of the space $E_{1}$ and the isomorphism between $E_{1}$ and $E \times \mathbb{R}^{n}$. For example, for systems of first order ordinary differential equations or differential equations with deviating argument the space $E_{1}$ is usually a space of absolutely continuous functions on an interval, while for systems of impulsive differential equations $E_{1}$ can be chosen as a space of piecewise absolutely continuous functions with fixed points of discontinuity (see the constructions of A. Anokhin in [1: Sections 6.3 and 6.4]). For more examples we refer to paper [3], where some general approaches to construct appropriate pairs of spaces $E_{1}$ and $E$ with $E_{1} \simeq E \times \mathbb{R}^{n}$ for many other important classes of functional-differential equations are considered (in particular, for equations "with quasi-derivatives" and for integro-differential equations over the whole real axis) and to [2] where such constructions are applied to various types of singular functional-differential equations.

Recall the following definition of a bifurcation point [11: $\S 56]$.

Definition 1. $\lambda_{1}^{\prime} \in \mathbb{R}$ is called a bifurcation point of problem (3) if in each neighborhood of $\lambda_{1}^{\prime}$ for any $\varepsilon>0$ there exists a value of the parameter $\lambda_{1}$ such that (3) admits a non-trivial solution $x \neq 0$ of norm $\|x\|_{E_{1}}<\varepsilon$.

Recall now some well-known facts from bifurcation theory. Consider our problem in the form of one equation

$$
L x=F\left(x, \lambda_{1}\right)
$$

where $x \in E_{1}, L: E_{1} \rightarrow E \times \mathbb{R}^{n}, L=\{\mathcal{L}, l\}$ and $F: E_{1} \times \mathbb{R} \rightarrow E \times \mathbb{R}^{n}, F=\{\mathcal{F}, \phi\}$ are linear and nonlinear operators, respectively, and $F(0, \mathbb{R})=0$. Assuming sufficient smoothness of $F$, namely that in some neighborhood of $x=0$ the representation

$$
F\left(x, \lambda_{1}\right)=F_{x}\left(0, \lambda_{1}\right) x+R\left(x, \lambda_{1}\right)
$$

is valid with $F_{x}\left(0, \lambda_{1}\right): E_{1} \rightarrow E \times \mathbb{R}^{n}$ being a linear and $R: E_{1} \times \mathbb{R} \rightarrow E \times \mathbb{R}^{n}$ a nonlinear operator containing higher-order terms in $x$ (in the sequel we make these conditions more 
concrete), one gets the well-known fact [11: Theorem 56.1] that all bifurcation points of (4) provide non-trivial solvability of the respective linearized problem

$$
L x=F_{x}\left(0, \lambda_{1}\right) x
$$

in other words, they are characteristic values of the latter. To answer the converse question, which of the characteristic values of the linearized equation (6) are the bifurcation points of the original nonlinear equation, one has available a lot of powerful statements like the M.A. Krasnosel'skii principle of index change and related theorems (see [4] and [10] for an overview of bifurcation theory). However, trying to apply even the simplest of such principles like the famous simple eigenvalue theorem to functional-differential equations, one often encounters the following obstacle: in general the linearized problem (6) can have a rather complicated structure, so it is rather difficult to examine even the existence of characteristic values, to say nothing of their multiplicities. Moreover, in certain problems $F_{x}\left(0, \lambda_{1}\right) x$ is not a linear operator, but only homogeneous of order 1 in $x$. This situation appears rather frequently when studying differential equations with "maxima" [17]. This could make the analysis of a linearized problem" (6) rather difficult. Nevertheless, one can have complete information on the structure of the left-hand part, i.e. on the operator $L$, which can help in solving the problem. This will be exploited in the sequel.

\section{Leray-Schauder degree theory for a class of mappings}

In what follows we will need a tool to study the solvability of systems of functional equations of the type

$$
\left\{\begin{array}{l}
\mathcal{F}(u, \lambda)=u \\
\mathbf{D}(u, \lambda)=0
\end{array}\right.
$$

where $u \in E_{0}, \lambda \in \mathbb{R}^{k}, \mathcal{F}: E_{0} \times \mathbb{R}^{k} \rightarrow E_{0}$ is a nonlinear operator, $\mathbf{D}: E_{0} \times \mathbb{R}^{k} \rightarrow \mathbb{R}^{k}$ is a nonlinear vector functional, and $E_{0}$ is a (infinite-dimensional) Banach space. For this purpose we apply the ordinary Leray-Schauder degree theory to mappings of the form

$$
\Psi(u, \lambda)=\left\{\begin{array}{c}
u-\mathcal{F}(u, \lambda) \\
\mathbf{D}(u, \lambda)
\end{array}\right\}
$$

Introduce the regions (open bounded subsets) $\Omega_{1} \subset E_{0}$ and $\Omega_{2} \subset \mathbb{R}^{k}$, and denote their boundaries by $\partial \Omega_{1}$ and $\partial \Omega_{2}$, respectively. Also, let $\Omega=\Omega_{1} \times \Omega_{2}$.

To apply the Leray-Schauder degree theory one needs the compactness property of $\Psi$, which is provided by assuming that $\mathcal{F}$ is a compact and continuous operator and $\mathbf{D}$ is a continuous vector functional, i.e. it maps bounded sets into bounded ones. The additional assumption of non-degeneracy of the mapping $\Psi$ on $\partial \Omega$ is enough to define correctly a topological degree $\operatorname{deg}(\Psi, \Omega, 0)$ satisfying all ordinary properties (see [15]). We say that the mapping $\Psi$ (the system (7)) is topologically non-trivial on the regions $\Omega_{1}$ and $\Omega_{2}$ (is of type $V\left(\Omega_{1}, \Omega_{2}\right)$, for short) if $\operatorname{deg}(\Psi, \Omega, 0) \neq 0$. Under some additional requirements on $\Psi$ one can calculate its topological degree in a rather simple way. 
Namely, consider the auxiliary finite-dimensional continuous vector field $\mathbf{D}_{0}: \mathbb{R}^{k} \rightarrow \mathbb{R}^{k}$ and let $|\cdot|$ be the norm in $\mathbb{R}^{k}$. To verify the topological non-triviality of a system (7) one can use the following result extending the analogous ones stated in $[18,21]$. These results probably originate in the theorem of J. Cronin [15] and constitute a common topological background, as mentioned in the introduction, for our approach and the coincidence degree of J. Mawhin [14].

Theorem 1. Let $\Omega_{1} \subset E_{0}$ be contractible, $\mathcal{F}$ be a compact and continuous operator and $\mathbf{D}$ be a continuous vector functional which maps bounded sets into bounded ones, while the following conditions hold:

(i) $\mathcal{F}\left(\partial \Omega_{1}, \mathrm{cl} \Omega_{2}\right) \subset \Omega_{1}$.

(ii) $0 \leq\left|\mathbf{D}(u, \lambda)-\mathbf{D}_{0}(\lambda)\right|<\left|\mathbf{D}_{0}(\lambda)\right|$ for all $u \in \operatorname{cl} \Omega_{1}$ and all $\lambda \in \partial \Omega_{2}$.

Then $\operatorname{deg}(\Psi, \Omega, 0)=\operatorname{deg}\left(\mathbf{D}_{0}, \Omega_{2}, 0\right)$. In particular, when the latter differs from zero, then the system of equations (7) has at least one solution $\left(u^{\prime}, \lambda^{\prime}\right) \in \Omega$. The set of such solutions can be approximated by the Galerkin numerical scheme applied to the system of equations (7).

Remark. In the sequel we will take $\mathbf{D}_{0}(\lambda)=\mathbf{D}(0, \lambda), \Omega_{1}=B_{\rho_{1}}(0) \subset E_{0}$ (the ball $\left.\|u\|_{E_{0}}<\rho_{1}\right)$ and $\Omega_{2}=B_{\rho_{2}}\left(\lambda^{*}\right) \subset \mathbb{R}^{k}$ (the ball $\left|\lambda-\lambda^{*}\right|<\rho_{2}$, where $\lambda^{*}$ is one of the roots of the system of algebraic equations $\mathbf{D}_{0}(\lambda)=0$ ). To verify that the system (7) is of type $V\left(\Omega_{1}, \Omega_{2}\right)$, it is enough (by Theorem 1 ) to study the solvability for a system of inequalities for $\rho_{1}>0$ and $\rho_{2}>0$ of the type

$$
\left\{\begin{array}{l}
U_{1}\left(\rho_{1}, \rho_{2}\right)<\rho_{1}, \\
U_{2}\left(\rho_{1}, \rho_{2}\right)<\rho_{2} .
\end{array}\right.
$$

Proof of Theorem 1. Contractibility of $\Omega_{1}$ implies the existence of a continuous map $h: \Omega_{1} \times[0,1] \rightarrow \Omega_{1}$, such that $h(0, u)=u, h(1, u)=u_{0}$ and $h(t, u) \in \Omega_{1}$ for all $(u, t) \in \Omega_{1} \times[0,1]$. Consider a homotopy

$$
\Psi_{t}(u, \lambda)=\left\{\begin{array}{c}
u-h(t, \mathcal{F}(u, \lambda)) \\
\mathbf{D}_{0}(\lambda)+(1-t)\left(\mathbf{D}(u, \lambda)-\mathbf{D}_{0}(\lambda)\right)
\end{array}\right\} \quad(t \in[0,1])
$$

Obviously, it is compact and according to conditions (i) and (ii) non-degenerate on $\partial \Omega$. Thus by the homotopy property of the degree and the product formula (see [11: Theorem 22.4])

$$
\operatorname{deg}(\Psi, \Omega, 0)=\operatorname{deg}\left(\Psi_{0}, \Omega, 0\right)=\operatorname{deg}\left(\Psi_{1}, \Omega, 0\right)=\operatorname{deg}\left(\mathbf{D}_{0}, \Omega_{2}, 0\right)
$$

The rest of the statement follows trivially from the basic properties of the LeraySchauder degree. 


\section{Linearization theorems}

Consider an equation of type (4) and assume that $L$ is a Fredholm operator of index zero with non-trivial kernel and adjoint kernel given by

$$
\operatorname{ker} L=\operatorname{span}\left\{\varphi_{1}, \ldots, \varphi_{k}\right\} \quad \text { and } \quad \operatorname{ker} L^{*}=\operatorname{span}\left\{\psi_{1}, \ldots, \psi_{k}\right\},
$$

respectively. Note that this is not a restriction; in practice one can provide such a condition even "artificially", e.g. when $L$ is continuously invertible and there exists a linear operator $L_{1}: E_{1} \rightarrow E \times \mathbb{R}^{n}$ such that $L+L_{1}$ is a Fredholm operator of index zero with non-trivial kernel, one simply considers the equation

$$
\left(L+L_{1}\right) x=F_{1}\left(x, \lambda_{1}\right)
$$

where $F_{1}\left(x, \lambda_{1}\right)=F\left(x, \lambda_{1}\right)+L_{1} x$. Furthermore, assume the operator $F_{x}\left(0, \lambda_{1}\right) x$ to be homogeneous of order 1 in $x$ (not necessarily additive). Now, following the technique presented in [21], we will look for bifurcation in the direction $\varphi_{1}$ by introducing into the problems (4) and (6) a substitution of variables

$$
x=\xi\left(u+\varphi_{1}+\sum_{i=2}^{k} \lambda_{i} \varphi_{i}\right),
$$

where $u \in E_{0}=E_{1} / \operatorname{kerL}$ and $\lambda_{i} \in \mathbb{R}$. Applying then the Lyapunov-Schmidt reduction procedure and assuming $\xi \neq 0$ one arrives at the auxiliary systems of branching equations with respect to the unknown elements $u \in E_{0}$ and $\lambda=\left\{\lambda_{1}, \ldots, \lambda_{k}\right\} \in \mathbb{R}^{k}$ written out below:

1. For the nonlinear problem (4):

$$
\left\{\begin{aligned}
\frac{1}{\xi} \hat{L}^{-1} F\left(\xi\left(u+\varphi_{1}+\sum_{i=2}^{k} \lambda_{i} \varphi_{i}\right), \lambda_{1}\right) & =u \\
\frac{1}{\xi}\left\langle F\left(\xi\left(u+\varphi_{1}+\sum_{i=2}^{k} \lambda_{i} \varphi_{i}\right), \lambda_{1}\right), \psi_{j}\right\rangle & =0 \quad(j=1, \ldots, k),
\end{aligned}\right.
$$

or, for short,

$$
\Psi(u, \lambda, \xi)=0 \quad \text { for } \quad \xi \neq 0 .
$$

2. For the linearized problem (6):

$$
\left\{\begin{aligned}
\hat{L}^{-1} F_{x}\left(0, \lambda_{1}\right)\left(u+\varphi_{1}+\sum_{i=2}^{k} \lambda_{i} \varphi_{i}\right) & =u \\
\left\langle F_{x}\left(0, \lambda_{1}\right)\left(u+\varphi_{1}+\sum_{i=2}^{k} \lambda_{i} \varphi_{i}\right), \psi_{j}\right\rangle & =0 \quad(j=1, \ldots, k),
\end{aligned}\right.
$$

or, for short,

$$
\Psi(u, \lambda, 0)=0
$$


where the mapping $\Psi(u, \lambda, \xi)$ is defined as

$$
\Psi(u, \lambda, \xi)=\Psi(u, \lambda, 0)+\frac{1}{\xi}\left\{\begin{array}{c}
-\hat{L}^{-1} R\left(\xi\left(u+\varphi_{1}+\sum_{i=2}^{k} \lambda_{i} \varphi_{i}\right), \lambda_{1}\right) \\
\left\langle R\left(\xi\left(u+\varphi_{1}+\sum_{i=2}^{k} \lambda_{i} \varphi_{i}\right), \lambda_{1}\right), \psi_{1}\right\rangle \\
\vdots \\
\left\langle R\left(\xi\left(u+\varphi_{1}+\sum_{i=2}^{k} \lambda_{i} \varphi_{i}\right), \lambda_{1}\right), \psi_{k}\right\rangle
\end{array}\right\}
$$

at $\xi \neq 0$ and

$$
\Psi(u, \lambda, 0)=\left\{\begin{array}{c}
u-\hat{L}^{-1} F_{x}\left(0, \lambda_{1}\right)\left(u+\varphi_{1}+\sum_{i=2}^{k} \lambda_{i} \varphi_{i}\right) \\
\left\langle F_{x}\left(0, \lambda_{1}\right)\left(u+\varphi_{1}+\sum_{i=2}^{k} \lambda_{i} \varphi_{i}\right), \psi_{1}\right\rangle \\
\vdots \\
\left\langle F_{x}\left(0, \lambda_{1}\right)\left(u+\varphi_{1}+\sum_{i=2}^{k} \lambda_{i} \varphi_{i}\right), \psi_{k}\right\rangle
\end{array}\right\}
$$

Here the parameter $\xi \neq 0$ is free and the operator $\hat{L}^{-1}: E_{1} \rightarrow E_{0}$ stands for the generalized inverse of $L$.

Note that a trick with a non-equivalent substitution and dividing by a parameter of branching equations is applied frequently in bifurcation theory, in particular in the proof of the simple eigenvalue theorem (see [5] and [15]). Nevertheless, our trick differs a bit from the one in the mentioned proof: we look for bifurcation in the direction of the element $\varphi_{1}$ of the kernel of $L$, and not in the direction of the kernel of $L-F_{x}\left(0, \lambda_{1}^{\prime}\right)$, where $\lambda_{1}^{\prime}$ is "suspected" for bifurcation. The reason is that we are supposed to know much about $L$ and almost nothing about $L-F_{x}\left(0, \lambda_{1}^{\prime}\right)$ (we even may be unaware of the location of $\lambda_{1}^{\prime}$ ).

We are now able to formulate the following linearization result generalizing [23: Theorem 3]. Denote $P_{1}: \lambda \in \mathbb{R}^{k} \mapsto \lambda_{1} \in \mathbb{R}$ and let cl stand for the closure.

Theorem 2. Assume that the operator $F$ is continuous and maps bounded sets into bounded ones, while $\hat{L}^{-1} F$ is compact, the representation (5) is valid with $F_{x}\left(0, \lambda_{1}\right) x$ homogeneous of order 1 in $x$, and that there exist regions $\Omega_{1} \subset E_{0}$ and $\Omega_{2} \subset \mathbb{R}^{k}$ such that the following conditions hold:

(i) $\left\|R\left(x, \lambda_{1}\right)\right\|_{E \times \mathbb{R}^{n}}=o\left(\|x\|_{E_{1}}\right)$ when $\|x\|_{E_{1}} \rightarrow 0$, uniformly with respect to $\lambda_{1} \in$ $P_{1}\left(\mathrm{cl} \Omega_{2}\right)$.

(ii) The system of equations (12) is of type $V\left(\Omega_{1}, \Omega_{2}\right)$.

Then the original equation (4) has in $P_{1}\left(\Omega_{2}\right)$ at least one bifurcation point which is also a characteristic value of $(6)$.

Proof. From the representation (10) it follows that $\xi \neq 0$ implies $x \neq 0$ (otherwise it would contradict the definition of a factor-space). Hence all solutions of (11) and (12) correspond to non-trivial solutions of the respective original equations (4) and (6). 
From condition (ii) it follows that $\operatorname{deg}\left(\Psi(\cdot, \cdot, 0), \Omega_{1} \times \Omega_{2}, 0\right) \neq 0$ and the system (12) is solvable in $\Omega_{1} \times \Omega_{2}$. Due to condition (i) and the principle of topological degree stability with respect to small perturbations of the map [11: Theorem 19.2] one has $\operatorname{deg}\left(\Psi(\cdot, \cdot, \xi), \Omega_{1} \times \Omega_{2}, 0\right) \neq 0$ for all $\xi$ from a small neighborhood of zero, and the system (11) is solvable for sufficiently small $\xi$. Let $T_{0}$ stand for the solution set of (12) in $\Omega_{1} \times \Omega_{2}$ and $T_{\xi}$ stand for that of (11). From the estimate

$$
\|\Psi(u, \lambda, \xi)\|_{E_{0} \times \mathbb{R}^{k}} \leq\|\Psi(u, \lambda, \xi)-\Psi(u, \lambda, 0)\|_{E_{0} \times \mathbb{R}^{k}}+\|\Psi(u, \lambda, 0)\|_{E_{0} \times \mathbb{R}^{k}}
$$

and the fact of uniform non-degeneracy of $\Psi$ on any closed bounded set where it is different from zero [11: Section 19.4] it follows that given any neighborhood $U$ of $T_{0}$ there is $\delta>0$ such that $T_{\xi} \subset U$ for all' $\xi$ with $|\xi|<\delta$. Thus, there is a sequence $\xi_{k} \rightarrow 0$ such that for the corresponding $\lambda_{1_{k}} \in P_{1}\left(T_{\xi_{k}}\right)$ we have $\lambda_{1_{k}} \rightarrow \lambda_{1}^{\prime} \in P_{1}\left(T_{0}\right)$. To show that $\lambda_{1}^{\prime}$ is a bifurcation point it is enough to note according to (10) that for the respective $x_{k}$ we have $x_{k} \rightarrow 0$

To analyze the above statement, consider the classical particular case that $F_{x}\left(0, \lambda_{1}\right)$ : $E_{1} \rightarrow E \times \mathbb{R}^{n}$ is a linear continuous operator, and hence, according to condition (i) of Theorem 2, the nonlinear operator $F$ is Fréchet differentiable in $x=0$. It is clear that $\Psi(u, \lambda, 0)=0$ if and only if $x \in \operatorname{ker}\left(L-F_{x}\left(0, \lambda_{1}\right)\right)$, where $x=x(u, \lambda)$ is defined by (10). We show then that for condition (ii) of Theorem 2 to be fulfilled it is necessary that for each characteristic value $\lambda_{1}^{\prime} \in P_{1}\left(\Omega_{2}\right)$ the above kernel is one-dimensional. In fact, otherwise we would have that there is a line $\left\{x_{1}+t x_{2} \in \operatorname{ker}\left(L-F_{x}\left(0, \lambda_{1}^{\prime}\right)\right): t \in \mathbb{R}\right\}$, which has a projection on $\operatorname{span} \varphi_{1}$ equal to one, and thus the $\operatorname{degree} \operatorname{deg}\left(\Psi, \Omega_{1} \times \Omega_{2}, 0\right)$ would be undefined. Therefore, it is clear that in this situation we deal with bifurcation from a "simple" characteristic value, and it is not difficult to see that the above degree is equal to the sum of jumps of the indices of $L-F_{x}\left(0, \lambda_{1}\right)$ on characteristic values $\lambda_{1}^{\prime} \in$ $P_{1}\left(\Omega_{2}\right)$. However, even in this case the advantage of our approach in comparison with the standard simple eigenvalue theorem is the fact that one does not need any explicit information on the location of characteristic values and on the kernel of $L-F_{x}\left(0, \lambda_{1}\right)$ but only uses the information on the operator $L$.

One should also emphasize that the above result opens the way for numerical treatment of the bifurcation problem (4). Namely, to seek bifurcation points on the interval $P_{1}\left(\Omega_{2}\right)$ which gives rise to branches of non-trivial solutions with the parametrization (10), one can under the conditions of Theorem 2 apply the Galerkin numerical scheme to the system of equations (11) when $\xi \rightarrow 0$.

Let us also mention that in bifurcation theory one frequently finds existence results for bifurcation points in an open set formulated in terms of non-triviality of a topological degree or other homotopy invariant over this set. In general, however, one tries then to localize the bifurcation points within this set. A related example, where the further localization is not easy, is provided in [9: Theorem 2.2]. At last, it is worth recalling that what we have done by means of the Lyapunov-Schmidt reduction and the LeraySchauder degree, is equivalent to the coincidence degree construction of $\mathrm{J}$. Mawhin (except that we deal here not with the coincidence degree of a map in $E_{1}$ but on a hyperplane of $E_{1}$ ).

An analogous assertion for the situation when $F_{x}\left(0, \lambda_{1}\right)$ is a linear operator can be proved by finite-dimensional reduction of system (12). 
Theorem 3. Assume that for the continuous operator $F$ the representation (5) is valid with $F_{x}\left(0, \lambda_{1}\right) x$ linear in $x$ and $\hat{L}^{-1}$ is continuous, and that there exist $\rho_{2}>0$ and $\lambda^{*} \in \mathbb{R}^{*}$ such that the following conditions hold:

(i) $\left\|R\left(x, \lambda_{1}\right)\right\|_{E \times \mathbb{R}^{n}}=o\left(\|x\|_{E_{1}}\right)$ when $\|x\|_{E_{1}} \rightarrow 0$, uniformly with respect to $\lambda_{1} \in$ $I_{1}$, where $I_{1}=\left[\lambda_{1}^{*}-\rho_{2}, \lambda_{1}^{*}+\rho_{2}\right]$.

(ii) The linear equation $u-\hat{L}^{-1} F_{x}\left(0, \lambda_{1}\right) u=v \quad\left(u \in E_{0}\right)$ admits for all $\lambda_{1} \in I_{1}$ an a priori estimate $\|u\|_{E_{0}} \leq C\|v\|_{E_{0}}$ with $C=C\left(\rho_{2}, \lambda_{1}^{*}\right)$, and there exists a $\lambda_{1}=\hat{\lambda}_{1} \in I_{1}$ such that this equation is solvable for all $v \in E_{0}$.

(iii) The vector field $\mathbf{X}(\lambda): \mathbb{R}^{k} \rightarrow \mathbb{R}^{k}$ is non-degenerate on the sphere $\partial B_{\rho_{2}}\left(\lambda^{*}\right)$, and its topological degree satisfies $\operatorname{deg}\left(\mathbf{X}, B_{\rho_{2}}\left(\lambda^{*}\right), 0\right) \neq 0$, where

$$
X_{j}(\lambda)=\left\langle F_{x}\left(0, \lambda_{1}\right)\left(I d_{E_{0}}+H^{-1}\left(\lambda_{1}\right) \hat{L}^{-1} F_{x}\left(0, \lambda_{1}\right)\right)\left(\varphi_{1}+\sum_{i=2}^{k} \lambda_{i} \varphi_{i}\right), \psi_{j}\right\rangle
$$

for $j=1, \ldots, k, H\left(\lambda_{1}\right)=I d_{E_{0}}-\hat{L}^{-1} F_{x}\left(0, \lambda_{1}\right)$ and $I d_{E_{0}}$ the identity in $E_{0}$.

Then the nonlinear equation (4) has at least one bifurcation point $\lambda_{1}^{\prime} \in\left(\lambda_{1}^{*}-\rho_{2}, \lambda_{1}^{*}+\right.$ $\left.\rho_{2}\right)$.

Proof. The first equation of system (12) is uniquely solvable with respect to the unknown $u$ for $\lambda \in \operatorname{cl} B_{\rho_{2}}$ :

$$
u=H^{-1}\left(\lambda_{1}\right) \hat{L}^{-1} F_{x}\left(0, \lambda_{1}\right)\left(\varphi_{1}+\sum_{i=2}^{k} \lambda_{i} \varphi_{i}\right)+o(1)
$$

as $\xi \rightarrow 0$. Note that $u(\lambda)$ is continuous due to the conditions of the theorem. Substituting the expression $u(\lambda)$ into the finite-dimensional part of the system of equations (12) we come to an equivalent system of algebraic equations. From condition (iii), using the principle of (finite-dimensional) topological degree stability with respect to small perturbations [11: Theorem 19.2], one yields the desired conclusion

The major shortcoming of the linearization Theorem 3 is that it requires to construct the expression for $\mathbf{X}(\lambda)$, that can be a rather hard job because of the necessity to invert the operator $H\left(\lambda_{1}\right)$. It is therefore helpful to reformulate this theorem so that its conditions become easier to verify. For this purpose we claim the following assertion.

Corollary 1. Let the conditions (i) and (ii) of the linearization Theorem 3 be satisfied and the finite-dimensional vector field $\mathrm{D}_{0}(\lambda)$ be non-degenerate on the sphere $\partial B_{\rho_{2}}\left(\lambda^{*}\right)$, while

$$
\operatorname{deg}\left(\mathbf{D}_{0}, B_{\rho_{2}}\left(\lambda^{*}\right), 0\right) \neq 0
$$

and

$$
\max _{\lambda_{1} \in\left\{\lambda_{i}^{*}-\rho_{2}, \lambda_{i}^{*}+\rho_{2}\right\}} \max _{\|u\|_{E_{0}} \leq \rho_{1}}\left|\Delta D\left(u, \lambda_{1}\right)\right|<\min _{\lambda \in \partial B_{\rho_{2}}\left(\lambda^{*}\right)}\left|\mathbf{D}_{0}(\lambda)\right|,
$$

where

$$
\rho_{1}=C\left(\rho_{2}, \lambda_{1}^{*}\right)\left\|\varphi_{1}+\sum_{i=1}^{k} \lambda_{i} \varphi_{i}\right\|_{E_{1}}
$$


and

$$
\left\{\begin{array}{rl}
D_{0_{j}}(\lambda) & =\left\langle F_{x}\left(0, \lambda_{1}\right)\left(\varphi_{1}+\sum_{i=2}^{k} \lambda_{i} \varphi_{i}\right), \psi_{j}\right\rangle \\
\Delta D_{j}\left(u, \lambda_{1}\right) & =\left\langle F_{x}\left(0, \lambda_{1}\right) u, \psi_{j}\right\rangle
\end{array} \quad(j=1, \ldots, k) .\right.
$$

Then the nonlinear equation (4) has at least one bifurcation point $\lambda_{1}^{\prime} \in\left(\lambda_{1}^{*}-\rho_{2}, \lambda_{1}^{*}+\rho_{2}\right)$.

\section{Branching equations for abstract functional-differential equations}

We turn now to bifurcation problems for an abstract functional-differential equation in the form (3) and derive for it the respective systems of branching equations (12) which we are going to analyze using the linearization theorems stated in the previous section in order to get bifurcation results. It is important to note that when constructing such systems we are rather free in the choice of the generalized inverse operator $\hat{L}^{-1}$ induced by the factorization of the space $E_{1}$ by $\operatorname{ker} L$. An appropriate choice of the latter could be rather essential in constructing systems of branching equations which are easy to study.

To explain the latter statement, we recall that our general idea is to get conditions for the existence of bifurcation points of (3) in terms of only some estimates, which at the same time should be sufficiently sharp to be sensible to the structure of the problem. These estimates are to be obtained by majorizing the branching equations. The problem is to choose the right space to make these estimates in. In fact, the original space $E_{1}$ is possibly not the most comfortable to work with, since, as one can see from the examples given in [1: Chapter 6] and in the papers cited in Section 2, it can have rather complicated structure depending on the class of problems under consideration. We prefer to work in $E$, for which we always choose a "simple" space (usually, just a Lebesgue space). Besides, we get then a certain standardization of the technique, since the choice of $E$ usually does not depend on the class of problems considered. We pass therefore from the system of equations in the space $E_{0} \times \mathbb{R}^{k}$ to the system of equations in $E \times \mathbb{R}^{n+k}$. A negative result consists in increasing the dimension of the finite-dimensional part of the system. Fortunately, in some cases (in which the right choice of the generalized inverse is most important), we are able to preserve this dimension and to work in $E \times \mathbb{R}^{k}$. As we see in the next section, this technique really leads to rather sharp estimates.

Instead of the standard constructions based on Schmidt's lemma [20], which.are usually more adapted to the study of semihomogeneous problems (i.e. $\phi \equiv 0$ ), we use here a construction based on the generalized Green operator that was suggested by L.F. Rakhmatullina in [16].

Suppose that $\mathcal{L}$ is a Fredholm operator of index ind $\mathcal{L}=n$ satisfying $\operatorname{dim} \operatorname{ker} \mathcal{L}=n$. Then the equation $\mathcal{L} x=f$ has for all $f \in E$ a solution $x \in E_{1}$. Assume that the 
homogeneous problem

$$
\left\{\begin{array}{l}
\mathcal{L} x=0 \\
l x=0
\end{array}\right.
$$

has $k>0$ linearly independent solutions $x_{1}, \ldots, x_{k}$. If $k<n$, then let

$$
X=\left\{x_{1}, \ldots, x_{k}, v_{1}, \ldots, v_{n-k}\right\}
$$

be a fundamental vector of the equation $\mathcal{L} x=0$ with $\left\langle v_{i}, l_{j}\right\rangle=\delta_{i j} \quad(i, j=1, \ldots, n-k)$ where $\delta_{i j}$ denotes the Kronecker delta. If $k=n$, then $X=\left\{x_{1}, \ldots, x_{k}\right\}$. Let $E_{1}$ be embedded into a Banach space $E_{2} \simeq E_{1} \times \mathbb{R}^{k}$, the respective isomorphism being determined by the map

$$
y \in E_{2} \longmapsto(\pi y, \rho y)=(x, \beta) \in E_{1} \times \mathbb{R}^{k},
$$

where the projector $\pi: E_{2} \rightarrow E_{1}$ and the vector functional $\rho: E_{2} \rightarrow \mathbb{R}^{k}$ satisfy $\rho \pi=0$. Then, the assertion is (see [1: Theorems 6.1.11 and 6.1.13]) that there is a linear extension $\hat{\mathcal{L}}: E_{2} \rightarrow E$ of the operator $\mathcal{L}$ and a vector functional $\hat{l}: E_{2} \rightarrow \mathbb{R}^{k}$ such that the problem

$$
\left\{\begin{array}{l}
\hat{\mathcal{L}} x=f \\
l x=\alpha, \\
\hat{l} x=\hat{\alpha}
\end{array}\right.
$$

admits a unique solution for each $f \in E$ and $\{\alpha, \hat{\alpha}\} \in \mathbb{R}^{n+k}$. In particular, if $\alpha=0$ and $\hat{\alpha}=0$, then the respective solution is given by $\tilde{x}=\hat{G} f \in E_{2}$, where $\hat{G}: E \rightarrow E_{2}$ is a Green operator for the above problem. Moreover, in this case the problem

$$
\left\{\begin{aligned}
\hat{\mathcal{L}} x & =f \\
l x & =\alpha,
\end{aligned}\right.
$$

is solvable if and only if $\alpha \in \mathbb{R}^{n}$ and $f \in E$ are connected by the relationships

$$
\begin{aligned}
\alpha_{n-k+i} & =\sum_{j=1}^{n-k} \alpha_{j}\left\langle v_{j}, l_{n-k+i}\right\rangle-\left\langle\hat{G} f, \rho_{i}\right\rangle & (i=1, \ldots, k ; k<n) \\
\alpha_{i} & =-\left\langle\hat{G} f, \rho_{i}\right\rangle & (i=1, \ldots, n ; k=n),
\end{aligned}
$$

and the solution $x \in E_{1}$ is given by

$$
x=G f+ \begin{cases}\sum_{i=1}^{k} \lambda_{i} x_{i}+\sum_{i=1}^{n-k} \alpha_{i} v_{i}, & \text { when } k<n, \\ \sum_{i=1}^{k} \lambda_{i} x_{i}, & \text { when } k=n,\end{cases}
$$

where $G=\pi \hat{G}$ is a generalized Green operator.

Assume now that in problem (3) the nonlinear operators $\mathcal{F}: E_{1} \times \mathbb{R} \rightarrow E$ and $\phi: E_{1} \times \mathbb{R} \rightarrow \mathbb{R}^{\mathbf{n}}$ admit the representations

$$
\begin{gathered}
\mathcal{F}\left(x, \lambda_{1}\right)=\mathcal{F}_{x}\left(0, \lambda_{1}\right) x+\mathcal{R}\left(x, \lambda_{1}\right), \\
\phi\left(x, \lambda_{1}\right)=\phi_{x}\left(0, \lambda_{1}\right) x+\varphi\left(x, \lambda_{1}\right),
\end{gathered}
$$


where $\mathcal{F}_{x}\left(0, \lambda_{1}\right) x$ and $\phi_{x}\left(0, \lambda_{1}\right) x$ are homogeneous of order 1 in $x$, and

$$
\left\{\begin{aligned}
\left\|\mathcal{R}\left(x, \lambda_{1}\right)\right\|_{E} & =o\left(\|x\|_{E_{1}}\right), \\
\left|\varphi\left(x, \lambda_{1}\right)\right| & =o\left(\|x\|_{E_{1}}\right),
\end{aligned} \quad \text { as }\|x\|_{E_{1}} \rightarrow 0\right.
$$

uniformly with respect to $\lambda_{1}$ from closed bounded sets. Consider a non-equivalent change of variables of type (10)

$$
x=\xi\left(u+x_{1}+\sum_{i=2}^{k} \lambda_{i} x_{i}\right)
$$

where $u \in E_{0}=E_{1} / \operatorname{ker} L$ and $\lambda_{i} \in \mathbb{R}$, and write out the respective systems of equations of type (12) with respect to the unknowns $u \in E_{0}$ and $\lambda \in \mathbb{R}^{k}$. For the case $k<n$ the latter has the form

$$
\left\{\begin{array}{c}
u=G \mathcal{F}_{x}\left(0, \lambda_{1}\right)\left(u+x_{1}+\sum_{i=2}^{k} \lambda_{i} x_{i}\right)+\sum_{j=1}^{n-k} v_{i} \phi_{x_{i}}\left(u+x_{1}+\sum_{i=2}^{k} \lambda_{i} x_{i}\right) \\
\phi_{x_{n-k+i}}\left(0, \lambda_{1}\right)\left(u+x_{1}+\sum_{j=2}^{k} \lambda_{j} x_{j}\right) \\
=-\left\langle\hat{G} \mathcal{F}_{x}\left(0, \lambda_{1}\right)\left(u+x_{1}+\sum_{j=2}^{k} \lambda_{j} x_{j}\right), \rho_{i}\right\rangle \\
+\sum_{j=1}^{n-k}\left\langle v_{j}, l_{n-k+i}\right) \phi_{x_{i}}\left(0, \lambda_{1}\right)\left(u+x_{1}+\sum_{j=2}^{k} \lambda_{j} x_{j}\right) \quad(i=1, \ldots, k),
\end{array}\right.
$$

while for the case $k=n$ the form of system (12) is

$$
\left\{\begin{array}{l}
u=G \mathcal{F}_{x}\left(0, \lambda_{1}\right)\left(u+x_{1}+\sum_{i=2}^{k} \lambda_{i} x_{i}\right), \\
\phi_{x_{i}}\left(0, \lambda_{1}\right)\left(u+x_{1}+\sum_{j=2}^{k} \lambda_{j} x_{j}\right)=-\left\langle\hat{G} \mathcal{F}_{x}\left(0, \lambda_{1}\right)\left(u+x_{1}+\sum_{j=2}^{k} \lambda_{j} x_{j}\right), \rho_{i}\right\rangle, \\
(i=1, \ldots, n) .
\end{array}\right.
$$

The linearization Theorem 2 for these cases would read in the following way.

Proposition 1. Assume that under the above conditions the systems (15) and (16) for $k<n$ and $k=n$, respectively, are of type $V\left(\Omega_{1}, \Omega_{2}\right)$ for some regions $\Omega_{1} \subset E_{0}$ and $\Omega_{2} \subset \mathbb{R}^{n}$. Then the set of bifurcation points of problem (3) inside the interval $P_{1}\left(\Omega_{2}\right)$ is non-empty. 
Sometimes it is not worth distinguishing a linear part $l$ of the vector functional, determining the boundary conditions of (3). Namely, consider a problem

$$
\left\{\begin{aligned}
\mathcal{L} x & =\mathcal{F}\left(x, \lambda_{1}\right), \\
\phi\left(x, \lambda_{1}\right) & =0
\end{aligned}\right.
$$

and suppose that it admits the trivial solution $x=0$, while conditions (13) hold with $\mathcal{F}_{x}\left(0, \lambda_{1}\right) x$ and $\phi_{x}\left(0, \lambda_{1}\right) x$ homogeneous of order 1 in $x$. Recall [1: Chapter 6] that the assumptions imposed on the operator $\mathcal{L}$ imply the existence of an isomorphism

$$
J:(z, \beta) \in E \times \mathbb{R}^{n} \longmapsto x=\hat{G} z+\hat{X} \beta \in E_{1}
$$

with the inverse

$$
J^{-1}: x \in E_{1} \longmapsto(z, \beta)=(\mathcal{L} x, l x) \in E \times \mathbb{R}^{n}
$$

where $\hat{G}: E \rightarrow E_{1}$ (a Green operator), $\hat{X}: \mathbb{R}^{n} \rightarrow E_{1}$ (a fundamental matrix) and $l: E_{1} \rightarrow \mathbb{R}^{n}$ are linear operators. Then, letting $k=n$ and $E_{0}=E_{1} / \operatorname{ker} \mathcal{L}$ and using the substitution

$$
x=\xi\left(u+x_{1}+\sum_{i=2}^{n} \lambda_{i} x_{i}\right),
$$

where the $x_{i}$ stand for the columns of the matrix $\hat{X}$, the respective "linearized" problem is a system of branching equations of form (12) with respect to the unknowns $u \in E_{0}$ and $\lambda \in \mathbb{R}^{n}$

$$
\left\{\begin{aligned}
\hat{G} \mathcal{F}_{x}\left(0, \lambda_{1}\right)\left(u+x_{1}+\sum_{i=2}^{k} \lambda_{i} x_{i}\right) & =u \\
\phi_{x}\left(0, \lambda_{1}\right)\left(u+x_{1}+\sum_{j=2}^{k} \lambda_{j} x_{j}\right) & =0
\end{aligned}\right.
$$

The linearization Theorem 2 can be easily extended to this case. The keypoint is that topological non-triviality of the latter system of equations implies the existence of bifurcation points of (3). It is probably easier to study instead the topological nontriviality of an equivalent system of equations with respect to the unknowns $z \in E$ and $\lambda \in \mathbb{R}^{n}$

$$
\left\{\begin{array}{c}
\mathcal{F}_{x}\left(0, \lambda_{1}\right)\left(\hat{G} z+x_{1}+\sum_{i=2}^{k} \lambda_{i} x_{i}\right)=z, \\
\vdots \\
\phi_{x}\left(0, \lambda_{1}\right)\left(\hat{G} z+x_{1}+\sum_{j=2}^{k} \lambda_{j} x_{j}\right)=0 .
\end{array}\right.
$$

Thus we come to the following version of the linearization Theorem 2 (or, equivalently, of Proposition 1).

Proposition 2. Let under the above conditions the system (18) be of type $V\left(\Omega_{1}, \Omega_{2}\right)$ for some regions $\Omega_{1} \subset E$ and $\Omega_{2} \subset \mathbb{R}^{n}$. Then the set of bifurcation points of the problem (17) inside the interval $P_{1}\left(\Omega_{2}\right)$ is non-empty. 


\section{Applications}

In this section we illustrate the above abstract technique in particular examples. First consider the following sample boundary value problem for a functional-differential equation with deviating argument

$$
\left\{\begin{aligned}
\ddot{x}+\left(\lambda_{1} f(t)+q(t)\right) x(h(t))=r(x) & (t \in[0, T]), \\
x(\tau) & =0 \quad(\tau \notin[0, T]), \\
\int_{0}^{T} \dot{x}(t) d t & =\phi_{1}(x) \\
\int_{0}^{T} \ddot{x}(t) d t+\lambda_{1} \int_{0}^{T} x(t) d g(t)+\lambda_{1}^{3} x(T) & =\phi_{2}(x)
\end{aligned}\right.
$$

where $T>0$ is given, $f, q \in L^{1}(0, T), h:[0, T] \rightarrow \mathbb{R}$ is measurable, $g$ has bounded variation on $[0, T], \lambda_{1}$ is a bifurcation parameter, $r: A C^{1,1} \rightarrow L^{1}(0, T)$ and $\phi_{1}, \phi_{2}$ : $A C^{1,1}(0, T) \rightarrow \mathbb{R}$ are nonlinear continuous operators satisfying $r(0)=0,\|r(x)\|_{1}=$ $o\left(\|x\|_{A C^{1,1}}\right)$ and $\phi_{i}(0)=0,\left|\phi_{i}(x)\right|=o\left(\|x\|_{A C^{1,1}}\right)(i=1,2)$ as $\|x\|_{A C^{1,1}} \rightarrow 0$. Let $\|\cdot\|_{p}$ denote the norm in $L^{p}(0, T)$. We assume that $r(\cdot)$ is compact. It is not quite easy to get some information on the structure of the spectrum of the respective linearized problem. However, using the developed technique we can find some intervals containing bifurcation points and applying the Galerkin numerical scheme to the systems of branching equations we can localize the bifurcation points in these intervals. In fact, recall that $A C^{1,1} \simeq L^{1}(0, T) \times \mathbb{R}^{2}$ and the isomorphism can be represented, for example, in the form

$$
(z, \beta) \in L^{1}(0, T) \times \mathbb{R}^{2} \longmapsto \int_{0}^{T} G_{0}(t, \tau) z(\tau) d \tau+\left(1-\frac{t}{T}\right) \beta_{1}+\frac{t}{T} \beta_{2} \in A C^{1,1},
$$

where

$$
G_{0}(t, \tau)= \begin{cases}\frac{(t-T) \tau}{T}, & \text { if } 0 \leq \tau \leq t<T \\ -\frac{t(T-\tau)}{T}, & \text { if } 0 \leq t \leq \tau \leq T\end{cases}
$$

is the usual Green function for a two-point boundary value problem

$$
\left\{\begin{aligned}
\ddot{x}(t) & =p(t) \\
x(0) & =0 \\
x(T) & =0
\end{aligned}\right.
$$

Let

$$
(\mathcal{L} x)(t)=\ddot{x}(t) \quad \text { and } \quad l x=\left\{\int_{0}^{T} \dot{x}(t) d t, \int_{0}^{T} \ddot{x}(t) d t\right\}
$$


Obviously, one has then (with the previous notation) $k=1<n=2, x_{1}(t)=1$ and $v_{1}(t)=t / T$. The system of branching equations of type (12) with respect to the unknowns $u \in E_{0}=A C^{1,1} / \operatorname{ker} L$ and $\lambda_{1} \in \mathbb{R}$ can be written in the form

$$
\left\{\begin{array}{c}
\int_{0}^{T} G_{0}(t, \tau)\left(\lambda_{1} f(\tau)+q(\tau)\right) S_{h}(u(\tau)+1) d \tau=u(t), \\
\quad \int_{0}^{T}\left(\lambda_{1} f(\tau)+q(\tau)\right) S_{h}(u(\tau)+1) d \tau \\
+\lambda_{1} \int_{0}^{T}(u(\tau)+1) d g(\tau)+\lambda_{1}^{3}(1+u(T))=0
\end{array}\right.
$$

where $S_{h}$ is an inner superposition operator [1: Chapter 1] defined formally by the expression

$$
\left(S_{h} x\right)(t)= \begin{cases}x(h(t)), & \text { if } h(t) \in[0, T], \\ 0, & \text { if } h(t) \notin[0, T] .\end{cases}
$$

Minding the isomorphism (20), after some simple transformations, we observe that system (21) is topologically non-trivial on a region of $E_{0} \times \mathbb{R}$ if and only if the following system with respect to the unknowns $z \in L^{1}(0, T)$ and $\lambda_{1} \in \mathbb{R}$ on the respective regions is so:

$$
\left\{\begin{array}{r}
\left(\lambda_{1} f(t)+q(t)\right)\left(1_{h}(t)+\left(\Lambda_{h} z\right)(t)\right)=z(t) \\
\int_{0}^{T}\left(\lambda_{1} f(\tau)+q(\tau)\right)\left(1_{h}(\tau)+\left(\Lambda_{h} z\right)(\tau)\right) d \tau \\
+\lambda_{1} \int_{0}^{T}\left(1_{h}(\tau)+\left(\Lambda_{h} z\right)(\tau)\right) d g(\tau)+\lambda_{1}^{3}=0
\end{array}\right.
$$

where

$$
\left(\Lambda_{h} z\right)(t)=S_{h} \int_{0}^{T} G_{0}(t, \tau) z(\tau) d \tau \quad \text { and } \quad 1_{h}(t)=\left(S_{h} 1\right)(t) .
$$

Consider now the auxiliary one-dimensional vector field

$$
D_{0}\left(\lambda_{1}\right)=\lambda_{1}^{3}+a_{11} \lambda_{1}+b_{1}
$$

where

$$
a_{11}=\int_{0}^{T} f(t) 1_{h}(t) d t+\int_{0}^{T} 1_{h}(t) d g(t) \quad \text { and } \quad b_{1}=\int_{0}^{T} q(t) 1_{h}(t) d t
$$


and denote

$$
\begin{aligned}
c_{f} & =\|f\|_{1} / T, \\
c_{q} & =\|q\|_{1} / T, \\
c_{h} & =\sup _{h(t) \in[0, T], \tau \in[0, T]}\left|G_{0}(h(t), \tau)\right|, \\
c_{g} & =\left(\operatorname{var}_{[0, T]} g(t)\right) / T .
\end{aligned}
$$

One can now proclaim the following simplest statement.

Proposition 3. Assume that there exist a number $\rho_{2}>0$ such that $D_{0}\left(-\rho_{2}\right)$. $D_{0}\left(\rho_{2}\right)<0$ and the system of inequalities

$$
\left\{\begin{array}{c}
T c_{h}\left(\rho_{2} c_{f}+c_{q}\right)<1, \\
\frac{T^{2} c_{h}\left(\rho_{2} c_{f}+c_{q}\right)\left(\rho_{2}\left(c_{f}+c_{g}\right)+c_{q}\right)}{1-T c_{h}\left(\rho_{2} c_{f}+c_{q}\right)}<d\left(\rho_{2}\right)
\end{array}\right.
$$

holds, where $d\left(\rho_{2}\right)=\min \left\{\left|D_{0}\left(-\rho_{2}\right)\right|,\left|D_{0}\left(\rho_{2}\right)\right|\right\}$. Then problem (19) admits at least one bifurcation point on the interval $\left(-\rho_{2}, \rho_{2}\right)$.

Proof. Let $\Omega_{1} \subset L^{1}(0, T)$ be the open ball $\|z\|_{1}<\rho_{1}$. By Theorem 1 , it is easy to verify that $(22)$ is of type $V\left(\Omega_{1},\left(-\rho_{2}, \rho_{2}\right)\right)$ whenever the system of inequalities

$$
\left\{\begin{array}{c}
T c_{h}\left(\rho_{2} c_{f}+c_{q}\right)\left(1+\bar{\rho}_{1}\right)<\bar{\rho}_{1}, \\
T\left(\rho_{2} c_{f}+c_{q}\right) \bar{\rho}_{1}+T \rho_{2} c_{g} \bar{\rho}_{1}<d\left(\rho_{2}\right)
\end{array}\right.
$$

holds, where $\bar{\rho}_{1}=c_{h} \rho_{1}$. Applying the principle topological degree invariance with respect to linear homeomorphic transformations of the map and the Leray-Schauder lemma [11: Theorem 7.3], one shows that there is a region $\Omega_{1}^{\prime} \in E_{0}$ such that $(21)$ is of type $V\left(\Omega_{1}^{\prime},\left(-\rho_{2}, \rho_{2}\right)\right)$. The assertion now follows from Proposition 1

We note that the conditions of the above proposition are always satisfied for suffciently large $\rho_{2}$ and sufficiently small $\|f\|_{1},\|q\|_{1}$ and $\operatorname{var}_{[0, T]} g(t)$, while the necessary smallness can be easily estimated from the system of inequalities in the statement. It is also worth mentioning that the above estimates are rather sharp in the sense that they involve also the information on the function $h(t)$. Furthermore, one easily proves by the same method that under appropriate choice of $a_{11}$ and $b_{1}$ the original problem admits three different intervals containing bifurcation points. The characteristic values of the linearized problem within such interval(s) can be found by applying the Galerkin method to the system of equations (22), while the branches of non-trivial solutions (and the bifurcation points) of the original problem are found by applying the Galerkin method to the respective system of equations of the form (11) when $\xi \rightarrow 0$.

The same method can be applied to analyze the solvability of bifurcation problems 
for differential equations with "maxima" like, for instance, the following sample one:

$$
\left\{\begin{aligned}
\ddot{x}+\left(\lambda_{1} f(t)+q(t)\right) \max _{\tau \in S(t)} x(\tau) & =r(x) \quad(t \in[0, T]), \\
x(\tau) & =0 \quad(\tau \notin[0, T]), \\
\int_{0}^{T} \dot{x}(t) d t & =\phi_{1}(x), \\
\int_{0}^{T} \ddot{x}(t) d t+\lambda_{1} \int_{0}^{T} x(t) d g(t)+\lambda_{1}^{3} x(T) & =\phi_{2}(x),
\end{aligned}\right.
$$

where $S:[0, T] \rightarrow \mathbb{R}$ is a given set-valued measurable map. Reiterating the above scheme for such an equation is a simple exercise. However, here the "linearized" problem contains an operator being positively homogeneous of order 1 but non-additive. The linearization theorem remains still valid for such operators. Clearly, the free parameter $\xi$ should not considered to be positive.

To understand better the results that can be obtained by the developed technique, we study the problem (1) announced in the introduction, preserving all above notation. Without loss of generality we require that $\int_{0}^{T} q(\tau) 1_{h}(\tau) d \tau=0$. A system of branching equations of type (22) with respect to the unknowns $z \in L^{1}(0, T)$ and $\lambda_{1} \in \mathbb{R}$ would read as

$$
\left\{\begin{aligned}
-\left(\lambda_{1}+q(t)\right)\left(\left(\Lambda_{h} z\right)(t)+1_{h}(t)\right) & =z(t), \\
\left.\int_{0}^{T}\left(\lambda_{1}+q(\tau)\right)\left(\Lambda_{h} z\right)(\tau)+1_{h}(\tau)\right) d \tau & =0 .
\end{aligned}\right.
$$

In the following proposition we assume for simplicity that $h(t) \in[0, T]$ a.e. on $[0, T]$.

Proposition 4. Let under the above conditions $c_{q} c_{h}<3-\sqrt{8}$. Then problem (1) has at least one bifurcation point $\lambda_{1}^{\prime} \in\left[-\rho_{2}^{*}, \rho_{2}^{*}\right]$, where

$$
\rho_{2}^{*}=\frac{1-3 c_{q} c_{h}-\left(c_{q}^{2} c_{h}^{2}-6 c_{q} c_{h}+1\right)^{\frac{1}{2}}}{4 c_{h}} .
$$

Proof. Setting $D_{0}\left(\lambda_{1}\right)=T \lambda_{1}$ and using Theorem 1 one observes that (24) is of type $V\left(\Omega_{1},\left(-\rho_{2}, \rho_{2}\right)\right)$ with $\Omega_{1}$ the same region as in the proof of Proposition 3 , whenever $\rho_{1}>0$ and $\rho_{2}>0$ satisfy the system of inequalities

$$
\left\{\begin{aligned}
c_{h}\left(\rho_{2}+c_{q}\right)\left(\bar{\rho}_{1}+1\right) & <\bar{\rho}_{1} \\
\bar{\rho}_{1}\left(\rho_{2}+c_{q}\right) & <\rho_{2}
\end{aligned}\right.
$$

where $\bar{\rho}_{1}=c_{h} \rho_{1}$. After a few straightforward transformations one arrives at the equivalent system of inequalities

$$
\left\{\begin{aligned}
c_{q} c_{h} & <3-\sqrt{8} \\
\frac{1-c_{q} c_{h}}{2}-\sqrt{\frac{\left(1-c_{q} c_{h}\right)^{2}}{4}-c_{q} c_{h}} & =\bar{\rho}_{1}^{*}<\bar{\rho}_{1}<1 \\
\frac{c_{q} \bar{\rho}_{1}}{1-\bar{\rho}_{1}} & <\rho_{2}<\frac{\bar{\rho}_{1}}{c_{h}\left(\bar{\rho}_{1}+1\right)}+c_{q},
\end{aligned}\right.
$$


wherefrom the correctness of the statement follows immediately if we choose the sequence of numbers $\rho_{2_{k}} \rightarrow \rho_{2}^{*}=\frac{c_{q} \bar{\rho}_{i}^{*}}{1-\bar{\rho}_{i}^{*}}$. As usual, applying Galerkin's numerical procedure to the respective system of equations of type (11) written out for the original problem (1) as $\xi \rightarrow 0$, we can find the bifurcation points of this problem inside the closed interval indicated in the statement of the theorem

We remark that $\rho_{2}^{*} \rightarrow 0$ as $c_{q} \rightarrow 0, T$ fixed, and $\rho_{2}^{*} \rightarrow 0$ as $T \rightarrow 0, c_{q}$ fixed.

\section{Conclusions}

In this section we summarize the principal features of the method developed to deal with bifurcation problems for functional-differential equations. The general scheme of analysis of such problems is divided essentially into three steps.

First the original problem is linearized and, combining the Lyapunov-Schmidt type reduction technique with some non-equivalent substitution of variables one comes to a system of branching equations, with the following two main features:

(a) Its solutions correspond to all solutions of the linearized problem, except the trivial one.

(b) The spectral parameter $\lambda_{1}$ becomes one of the unknowns of the system.

Then the latter system is analyzed by means of the Leray-Schauder degree theory (e.g., using Theorem 1). If it happens to be topologically non-trivial on some regions, then the linearization Theorem 2 asserts the existence of bifurcation points in the respective intervals of the real axis. Thus the existence (and location) of intervals containing bifurcation points is usually given only in terms of estimates for the linearized problem. We emphasize that by this moment no information on the non-linearities are used.

At last, to select bifurcation points among the characteristic values of the linearized problem found inside the intervals, one should apply the Galerkin numerical technique to the systems of branching equations related to the original problem, whereby the information on nonlinear terms is used implicitly.

One should be aware of a considerable freedom in constructing the systems of branching equations. This refers first of all to the choice of the "model" operator $L=\{\mathcal{L}, l\}$ as a starting point for the Lyapunov-Schmidt reduction. Moreover, if the dimension of its kernel is more than one, then there are several possibilities to choose non-equivalent substitutions that naturally correspond to the parametrization of solution branches. There is also an important freedom of the choice of the generalized inverse $\hat{L}^{-1}$ (or, in other words, the generalized Green operator $G$ ) corresponding to the factorization of the original function space $E_{1}$ by ker $L$. This combined with the appropriate choice of an isomorphism between $E_{1}$ and $E \times \mathbb{R}^{n}$ adapted to the problem under consideration leads to simple systems of branching equations and to estimates, which are usually not "rough".

At last one should mention that the price paid for the generality of the method is quite high: in general, it does not allow to localize all bifurcation points, but only points 
outside of some interval. Moreover, in the most frequent case when $F_{x}\left(0, \lambda_{1}\right)$ is linear this method gives only the intervals containing "simple" characteristic values, which leads to analogies of the simple eigenvalue theorem. Its principal advantage is that it does not require much knowledge on the structure of the solution set of the linearized problem reducing the study to the search for simple estimates. In addition, it also works in some practically important situations when $F_{x}\left(0, \lambda_{1}\right) x$ is homogeneous of order 1 but non-additive.

\section{References}

(1] Azbelev, N. V., Maksimov, V. P. and L. F. Rakhmatullina: Introduction to the Theory of Functional Differential Equations (in Russian). Moscow: Nauka 1991; Engl. transl. of the first part: Introduction to the Theory of Linear Functional Differential Equations. World Federation Publishers 1996.

[2] Azbelev, N. V.: The current status and trends to development of theory of functionaldifferential equations (in Russian). Izvestiya Vuzov: Matematika 38 (1994)6, 8 - 19; Engl. transl.: Russian Math. 38 (1994)6, 6 - 17.

[3] Azbelev, N. V. and G. G. Islamov: On the theory of abstract linear equation (in Russian). In: Funct. Diff. Equations. Perm: Polytechnical Institute 1989, $15-27$.

[4] Chow, S. and J. K. Hale: Methods of Bifurcation Theory (Grundlegen der Mathematischen Wissenschaften). Berlin - New York: Springer-Verlag 1982.

[5] Crandall, M. G. and P. H. Rabinowitz: Bifurcation from simple eigenvalues. J. Funct: Anal. 8 (1971), $321-340$.

[6] Fitzpatrick, P.: The parity as an invariant for detecting bifurcation of the zeroes of one parameter families of nonlinear Fredholm maps. Lect. Notes Math. 1537 (1993), $74-$ 142.

[7] Fitzpatrick, P. M. and J. Pejsachowicz: The fundamental group of the space of linear Fredholm operators and the global analysis of semilinear equations. Contemporary Math. 72 (1988), $47-88$.

[8] Furi, M., Martelli, M. and M. P. Pera: General bifurcation theory: Local results and applications. In: Proceedings of the Conference on "Differential Equations and Applications to Biology and to Industry" (eds.: K. Cooke et al.). London: World Scientific 1996, pp. $101-115$.

[9] Furi, M. and M. P. Pera: A continuation principle for periodic solutions of forced motion equations on manifold and applications to bifurcation theory. Pac. J. Math. 160 (1993), $219-244$.

[10] Izé, J.: Topological bifurcation. In: Topological Nonlinear Analysis: Degree, Singularity, and Variations (Progress in Nonlinear Differential Equations and their Applications: Vol. 15; eds.: M. Matzeu and A. Vignoli). Boston: Birkhäuser Verlag 1995, pp. $341-463$.

[11] Krasnosel'skii, M. A. and P. P. Zabreiko: Geometrical Methods of Nonlinear Analysis (in Russian). Moscow: Nauka 1975; Engl. transl.: Berlin: Springer-Verlag 1984.

[12] Maksimov, V. P. and A. N. Rumyantsev: Constructive methods in the theory of functional differential equations and their computer aided implementation. Funct. Diff. Equ. 4 (1997) (to appear).

[13] Martelli, M.: Continuation principles and boundary value problems. Lect. Notes Math. 1537 (1993), $32-73$. 
[14] Mawhin, J.: Topological degree and boundary value problems for nonlinear differential equations. Lect. Notes Math. 1537 (1993), 74 - 142.

[15] Nirenberg, L.: Topics in Nonlinear Functional Analysis. New York: Courant Inst. Math. Sci. 1974.

[16] Rakhmatullina, L. F.: Generalized Green's operator of an overdetermined boundary value problem for linear functional-differential equation (in Russian). Izvestiya Vuzov: Matematika 5 (1993), 95 - 100.

[17] Stepanov, E.: On solvability of some boundary value problems for differential equations with maxima. Top. Meth. Nonlin. Anal. (to appear).

[18] Stepanov, E.: Topological degree for systems of functional equations arizing in control theory. Funct. Diff. Equ. 3 (1995), $239-255$.

[19] Stepanov, E. and S. A. Vavilov: Turning points and eigenvalue problems. J. Math. Anal. Appl. 199 (1996), $699-727$.

[20] Vainberg, M. M. and V. A. Trenogin: The Theory of Branching of Solutions of Nonlinear Equations (in Russian). Moscow: Nauka 1969; Engl. transl.: Groningen: WoltersNoordhoff 1974.

[21] Vavilov, S. A.: Nontrivial solutions of some classes of operator equations (in Russian). Dokl. Akad. Nauk (Russian) 331 (1993), 7 - 10; Engl. transl. in: Russian Acad. Sci. Dokl. Math. 48 (1994), $4-9$.

[22] Vavilov, S. A.: A method of studying the existence of nontrivial solutions to some classes of operator equations with an application to resonance problems in mechanics. J. Nonlin. Anal. - Theory, Meth., Appl. 24 (1995), 747 - 764.

[23] Vavilov, S. A. and E. O. Stepanov: On applicability of the linearization method to compute bifurcation points for a class of operator equations (in Russian). Dokl. Akad. Nauk (Russian) (to appear).

Received 08.07.1996; in revised form 25.04.1997 